\title{
Optimal cut-off points after a daily meal corresponding to fasting goal levels of LDL-C and non-HDL-C in Chinese patients with coronary heart disease
}

\section{Li-Ling Guo}

Second Xiangya Hospital

Yan-qiao Chen

Second Xiangya Hospital

Qiu-zhen Lin

Second Xiangya Hospital

Feng Tian

Second Xiangya Hospital

Qun-Yan Xiang

Second Xiangya Hospital

Li-yuan Zhu

Second Xiangya Hospital

Ling Liu ( $\nabla$ feliuling@csu.edu.cn )

Second Xiangya Hospital https://orcid.org/0000-0001-6979-701X

Jin Xu

Second Xiangya Hospital

\section{Research}

Keywords: Coronary heart disease, Non-fasting, Low-density lipoprotein cholesterol, Non-high-density lipoprotein cholesterol, Cut-off points, Statins

Posted Date: January 15th, 2020

DOl: https://doi.org/10.21203/rs.2.20892/v1

License: (c) (1) This work is licensed under a Creative Commons Attribution 4.0 International License. Read Full License 


\section{Abstract}

Background: Although the detection of non-fasting blood lipids has been recommended in patients with coronary heart disease (CHD), the non-fasting cut-off points corresponding to the fasting goals of LDL$\mathrm{C}<1.8 \mathrm{mmol} / \mathrm{L}$ and non-HDL-C $<2.6 \mathrm{mmol} / \mathrm{L}$, respectively, have not been explored.

Methods: This study enrolled 397 inpatients with CHD. One hundred and ninety-seven patients took statins for $<1$ month $(\mathrm{m})$ or did not take any statin before admission (i.e. CHD1 group), while 204 patients took statins for $\geq 1 \mathrm{~m}$ before admission (i.e. CHD2 group). Blood lipid levels were measured at 0 $\mathrm{h}, 2 \mathrm{~h}$, and $4 \mathrm{~h}$ after a daily breakfast.

Results: Non-fasting low-density lipoprotein cholesterol (LDL-C) and non-high-density lipoprotein cholesterol (non-HDL-C) levels significantly decreased after a daily meal $(P<0.05)$. Both fasting and nonfasting LDL-C or non-HDL-C levels were significantly lower in the CHD2 group. The percent attainment of $\mathrm{LDL}-\mathrm{C}<1.8 \mathrm{mmol} / \mathrm{L}$ at $2 \mathrm{~h}$ or $4 \mathrm{~h}$ after a daily breakfast was significantly higher than that of its fasting point $(P<0.05)$, whereas that of non-HDL-C $<2.6 \mathrm{mmol} / \mathrm{L}$ was significantly higher only at $4 \mathrm{~h}(\mathrm{P}<0.05)$. Analysis of C-statistic showed that non-fasting cut-off points for LDL-C and non-HDL-C were $1.5 \mathrm{mmol} / \mathrm{L}$ and $2.4 \mathrm{mmol} / \mathrm{L}$, corresponding to their fasting goal levels of $1.8 \mathrm{mmol} / \mathrm{L}$ and $2.6 \mathrm{mmol} / \mathrm{L}$, respectively. When postprandial LDL-C and non-HDL-C goal attainments were re-evaluated by non-fasting cut-off points, there were no significant differences in percent attainment between fasting and non-fasting states.

Conclusions: Determination ofnon-fasting cut-off points is important to evaluate the efficacy of cholesterol-lowering therapy if blood lipids are detected after a daily meal.

\section{Introduction}

Elevated cholesterol level is an independent risk factor for coronary heart disease (CHD). To reduce the risk of ischemic events, fasting level of low-density lipoprotein cholesterol (LDL-C) should be controlled to $<1.8 \mathrm{mmol} / \mathrm{L}$ as the primary target, then that of non-high-density lipoprotein cholesterol (non-HDL-C) should be $<2.6 \mathrm{mmol} / \mathrm{L}$ as the secondary target of cholesterol control [1-5]. It is increasingly believed that atherosclerosis is a postprandial phenomenon because, at least with respect to lipids, we are in the postprandial phase for the most part of the day [6]. Considering that either fasting or non-fasting (i.e. postprandial) LDL-C level has a similar predictive value for all-cause death and cardiovascular death [7, 8], non-fasting lipids detection at a random time-point within at least $8 \mathrm{~h}$ after a daily meal has been recommended in the primary and secondary prevention against CHD [9-12]. However, both LDL-C and non-HDL-C levels show a tendency of decrease in the non-fasting state [13-15]. Moreover, there are only fasting cholesterol-lowering targets but not non-fasting ones in the published guidelines [1-4, 9-12]. It is uncertain whether these fasting targets are applicable to assessing cholesterol control as well as how to evaluate it in the non-fasting stage. 
Recently, we observed more substantial reductions in LDL-C and non-HDL-C levels in Chinese subjects with $\mathrm{CHD}$ at $2 \mathrm{~h}$ and $4 \mathrm{~h}$ after a daily breakfast $[13,16]$, appearing to be greater than those reported in large-scale clinical studies conducted in other countries $[14,15,17,18]$,although the potential cause remains uncertain. Additionally, it was proposed that non-HDL-C level may be a better prognostic factor than LDL-C level to evaluate the risk of future cardiovascular events [19-24]. Furthermore, non-fasting fluctuation of non-HDL-C level seem to be smaller than that of LDL-C [24]. Nevertheless, there have been no studies comparing the goal attainment of LDL-C with that of non-HDL-C in the non-fasting state.

Therefore, this study aimed to compare the percent attainments of fasting and non-fasting LDL-C and non-HDL-C reaching their fasting targets in CHD patients receiving short-term statins therapy. Furthermore, analysis of c-statistic or receiver operating characteristic curve (ROC) analysis was used to determine the non-fasting cut-off points corresponding to their fasting targets, and the percent attainments of non-fasting LDL-C and non-HDL-C were re-evaluated according to the non-fasting cut-off points.

\section{Methods}

\subsection{Study population}

From March 2017 to July 2019, 397 inpatients with CHD were enrolled from the Department of Cardiovascular Medicine of the Second Xiangya Hospital, Central South University. A total of 193 patients took statins $<1$ month $(\mathrm{m})$ or did not take any statin before admission (i.e. CHD1 group), and 204 patients took statins $\geq 1 \mathrm{~m}$ before admission (i.e. CHD2 group). CHD was defined as a history of myocardial infarction and/or angiographically proven coronary atherosclerosis in patients with angina pectoris.

The study was approved by the Ethics Committee of the Second Xiangya Hospital of Central South University and conformed to the ethical guidelines of the 1975 Declaration of Helsinki as reflected in a priori approval. Informed consent was obtained from all participants.

\subsection{Specimen collection}

All enrolled participants ate breakfast between 7 a.m. and 8 a.m. according to their regular diets after overnight fast for at least $8 \mathrm{~h}$. Their breakfasts were purchased from the various cafeterias in the hospital or were brought from home, rich in high-carbohydrate content, such as steamed bread, noodles, vermicelli, rice porridge, and others. Venous blood samples were collected before $(0 \mathrm{~h})$ and at $2 \mathrm{~h}$ and $4 \mathrm{~h}$ after breakfast.

\subsection{Determination of blood lipid levels}

Serum total cholesterol (TC) and triglyceride (TG) levels were measured using automated enzymatic assays. Serum high-density lipoprotein cholesterol (HDL-C) and LDL-C levels were measured using a chemical masking method [25]. All measurements, including that of albumin, were carried out on a fully 
automatic biochemical analyser (Hitachi 7170A, Hitachi Inc., Tokyo, Japan) and performed by a specialist who was unaware of the details of the study [26]. Non-HDL-C = TC - (HDL-C).

\subsection{Statistical analysis}

Data were analysed using SPSS version 19.0. (IBM Corp., Armonk, NY, USA) and Prism 6.0 (GraphPad Inc., San Diego, CA, USA). Quantitative variables were expressed as mean \pm standard deviation, and qualitative variables were expressed as numbers and percentages. The unpaired Student's t-test and chisquare test were used to analyse quantitative and qualitative variables, respectively. The optimal cut-off points for fasting LDL-C $(1.8 \mathrm{mmol} / \mathrm{L})$ and non-HDL-C $(2.6 \mathrm{mmol} / \mathrm{L})$ were determined using receiver operating characteristic (ROC curve) analysis. Based on the ROC curve, values determined using Youden analysis were used as cut-off points. All tests were two-tailed, and $\mathrm{P}<0.05$ was considered statistically significant.

\section{Results}

\subsection{Clinical characteristics and fasting blood lipids in two CHD groups}

The baseline characteristics of the CHD patients are shown in Table 1. Both groups were similar in terms of age, sex, body mass index, percentages of hypertension, current smoking, and diabetes mellitus. There were $56.5 \%$ patients taking statins $<1 \mathrm{~m}$ and $43.5 \%$ patients without statins treatment before admission in CHD1 group. Fasting serum levels of TC, LDL-C, and non-HDL-C in CHD2 group were significantly lower than those in CHD1 group $(P<0.05)$. The differences in fasting serum TG and HDL-C levels between the groups did not differ significantly (Table 1 ). 
Baseline characteristics of the study population.

\begin{tabular}{|c|c|c|}
\hline & \multicolumn{2}{|c|}{ CHD1 $(n=193)$ CHD2(n= 204ロ } \\
\hline $\begin{array}{l}\text { Age, y } \\
\text { Men, } \mathrm{n}(\%) \\
\text { BMI, kg/m2 } \\
\text { Hypertension, } \mathrm{n}(\%) \\
\text { current smoking, } \mathrm{n}(\%) \\
\text { DM, } \mathrm{n}(\%) \\
\text { taking statins, } \mathrm{n}(\%) \\
\text { statins } \geq 1 \mathrm{~m} \\
\text { statins }<1 \mathrm{~m} \\
\text { no stains, } \mathrm{n}(\%) \\
\mathrm{TG}, \mathrm{mmol} / \mathrm{L} \\
\mathrm{TC}, \mathrm{mmol} / \mathrm{L} \\
\mathrm{HDL}-\mathrm{C}, \mathrm{mmol} / \mathrm{L} \\
\text { LDL-C, mmol/L } \\
\text { non-HDL-C, mmol/L }\end{array}$ & $\begin{array}{l}60.3 \pm 9.3 \\
156(80.8) \\
24.9 \pm 3.0 \\
141(73.1) \\
110(57.0) \\
51(26.4) \\
0 \\
109(56.5) \\
84(43.5) \\
1.94 \pm 1.46 \\
4.10 \pm 1.04 \\
1.01 \pm 0.25 \\
2.57 \pm 0.90 \\
3.09 \pm 0.96\end{array}$ & $\begin{array}{l}62.0 \pm 8.7 \\
157(77.0) \\
24.9 \pm 3.0 \\
152(74.5) \\
103(50.5) \\
67(32.8) \\
204(100) * \\
0 \star \\
0^{\star} \\
1.74 \pm 1.10 \\
3.78 \pm 1.00^{\star} \\
1.01 \pm 0.24 \\
2.31 \pm 0.88^{\star} \\
2.77 \pm 0.97^{\star}\end{array}$ \\
\hline \multicolumn{3}{|c|}{$\begin{array}{l}\text { Abbreviations: CHD1 group: CHD patients taking stains }<1 \mathrm{~m} \text { and without stains treatment before } \\
\text { admission. CHD2 group: CHD patients taking stains } \geq 1 \mathrm{~m} \text { before admission. BMI, body mass index; } \\
\text { DM, diabetes mellitus; TG, triglyceride; TC, total cholesterol; HDL-C, high-density lipoprotein } \\
\text { cholesterol; LDL-C, low-density lipoprotein. * } \mathrm{P}<0.05 \text { when compared with CHD1 group. }\end{array}$} \\
\hline
\end{tabular}

\subsection{Comparison of changes in non-fasting blood lipids in the two groups}

Levels of TC, LDL-C, HDL-C, and non-HDL-C decreased significantly at $2 \mathrm{~h}$ and $4 \mathrm{~h}$ after a daily breakfast, while those of TG increased significantly at $4 \mathrm{~h}$ in the two groups and at $2 \mathrm{~h}$ in $\mathrm{CHD} 2$ group $(\mathrm{P}<0.05)$. Non-fasting levels of TC, LDL-C, and non-HDL-C in CHD2 group were significantly lower than those in CHD1 group $(P<0.05)$ (Fig. 1A-C). However, the differences in non-fasting levels of TG and HDL-C between the groups were not statistically significant (Fig. 1D, E).

\subsection{Non-fasting reductions in LDL-C and non-HDL-C levels in the two groups}

When the data at $2 \mathrm{~h}$ and $4 \mathrm{~h}$ after a daily meal were pooled as whole non-fasting data for further analysis, non-fasting reductions in LDL-C were $0.47 \mathrm{mmol} / \mathrm{L}$ and $0.46 \mathrm{mmol} / \mathrm{L}$, and non-HDL-C were $0.26 \mathrm{mmol} / \mathrm{L}$ and $0.24 \mathrm{mmol} / \mathrm{L}$ in CHD1 and CHD2 groups, respectively. The percentages of reduction in LDL-C were $17.1 \%$ and $18.5 \%$, and $7.2 \%$ and $7.7 \%$ in non-HDL-C in CHD1 and CHD2 groups, respectively.

There were no significant differences in the absolute reduction or percentage of reduction in LDL-C or non-HDL-C level between the groups. However, non-fasting reductions in LDL-C were greater than those of non-HDL-C (Fig. 2A, 2B).

Non-fasting albumin levels were measured in 89 patients among all CHD patients. There was no significant change in the albumin level after a daily breakfast (Fig. 2C), whereas the postprandial LDL-C level dropped significantly $(P<0.05)$ (Supplement: Table 1). 


\subsection{Evaluating goal attainments of LDL-C and non-HDL-C according to various targets in CHD2 group}

Considering the effect of significant reduction in LDL-C or non-HDL-C level after a daily meal on the nonfasting evaluation of goal attainment, the cut-off points in non-fasting state corresponding to fasting LDL-C goal $<1.8 \mathrm{mmol} / \mathrm{L}$ and non-HDL-C goal $<2.6 \mathrm{mmol} / \mathrm{L}$ were determined by ROC analysis. Data at $2 \mathrm{~h}$ and $4 \mathrm{~h}$ after a daily breakfast were pooled as the postprandial data for a whole analysis. ROC analysis showed that non-fasting cut-off points for LDL-C and non-HDL-C were $1.5 \mathrm{mmol} / \mathrm{L}$ and $2.4 \mathrm{mmol} / \mathrm{L}$, corresponding to their fasting goal levels of $1.8 \mathrm{mmol} / \mathrm{L}$ and $2.6 \mathrm{mmol} / \mathrm{L}$, respectively (Figure. 3A, B).

According to the recommended targets of LDL-C and non-HDL-C in the 2016 Chinese guidelines [2], the percent attainment of $\mathrm{LDL}-\mathrm{C}<1.8 \mathrm{mmol} / \mathrm{L}$ in the fasting state was significantly lower than that of nonHDL-C $<2.6 \mathrm{mmol} / \mathrm{L}(35.3 \%$ vs $53.8 \%, \mathrm{P}<0.001)$. After a daily breakfast, the percent attainment of nonHDL-C was higher than that of LDL-C at each non-fasting time point; however, the difference was not statistically significant. The percent attainment of LDL-C at $2 \mathrm{~h}$ or $4 \mathrm{~h}$ was significantly higher than its fasting value $(P<0.05)$, while only that of non-HDL-C at $4 \mathrm{~h}$ was significantly higher than its fasting value $(P<0.05)$. The percent attainments of LDL-C and non-HDL-C simultaneously reaching their targets were very close to that of LDL-C not only in the fasting state but also in the non-fasting state (Fig. $3 \mathrm{C}$ ).

According to the non-fasting cut-off points, the percent attainment of LDL-C $<1.5 \mathrm{mmol} / \mathrm{L}$ at $2 \mathrm{~h}$ or $4 \mathrm{~h}$ was $28.9 \%$ or $34.3 \%$, which was close to the percent attainment of $L D L-C<1.8 \mathrm{mmol} / \mathrm{L}$ in the fasting state. Similar phenomenon was found for non-HDL-C, i.e., the percent attainment of non-HDL-C < $2.4 \mathrm{mmol} / \mathrm{L}$ at $2 \mathrm{~h}$ or $4 \mathrm{~h}$ was close to the percent attainment of $L D L-C<1.8 \mathrm{mmol} / \mathrm{L}$ in the fasting state. Moreover, the percent attainment of LDL-C $<1.5 \mathrm{mmol} / \mathrm{L}$ was significantly lower than that of non-HDL-C $<$ $2.4 \mathrm{mmol} / \mathrm{L}$ at $2 \mathrm{~h}$ or $4 \mathrm{~h}$ (Fig. 3D).

According to the 2019 European guidelines, a stricter LDL-C goal $<1.4 \mathrm{mmol} / \mathrm{L}$ has been recommended for CHD patients [27]. However, there were only 22 patients (10.8\%) with fasting LDL-C $<1.4 \mathrm{mmol} / \mathrm{L}$ in $\mathrm{CHD} 2$ group. This number of patients was too small to conduct further analysis to determine the nonfasting cut-off point corresponding to fasting LDL-C $<1.4 \mathrm{mmol} / \mathrm{L}$.

\section{Discussion}

In this study, we found that when LDL-C goal $<1.8 \mathrm{mmol} / \mathrm{L}$ and non-HDL-C goal $<2.6 \mathrm{mmol} / \mathrm{L}$ were used for evaluating cholesterol control in Chinese CHD patients after short-term statins treatment, the target percentage attainment in the non-fasting state was significantly higher than that of the fasting state, suggesting that fasting targets were not suitable for the evaluation of postprandial goal attainment. To our knowledge, this is the first investigation using ROC analysis to determine the non-fasting LDL-C and non-HDL-C cut-off points corresponding to the fasting LDL-C and non-HDL-C target levels of $1.8 \mathrm{mmol} / \mathrm{L}$ and $2.6 \mathrm{mmol} / \mathrm{L}$, respectively. Notably, according to the non-fasting cut-off points, the non-fasting goal 
attainment of LDL-C or non-HDL-C was close to its fasting value evaluated by LDL-C goal $<1.8 \mathrm{mmol} / \mathrm{L}$ or non-HDL-C goal $<2.6 \mathrm{mmol} / \mathrm{L}$. This suggests that lower non-fasting targets could be needed to evaluate the efficacy of cholesterol-lowing therapy in the non-fasting state, particularly when fasting blood lipids are unavailable and the percentage reduction of LDL-C cannot be determined due to a lack of baseline non-fasting levels before treatment.

There are two targets to evaluate the efficacy of cholesterol-lowering treatment in CHD patients. First, LDL-C should achieve $\mathrm{a} \geq 50 \%$ reduction from baseline or a goal $<1.8 \mathrm{mmol} / \mathrm{L}$ according to the European and American guidelines [3,4], similar to the 2016 Chinese guidelines [2]. These recommendations refer only to cholesterol control in the fasting state. In this study, the goal attainment of LDL-C reduction $\geq 50 \%$ could not be evaluated because the baseline fasting or non-fasting LDL-C levels before treatment could not be obtained in most patients in the CHD2 group. Under these circumstances, a physician can only make clinical judgments based on LDL-C goal levels. A considerable number of CHD patients from other locations visit physicians but forget to remain in a fasting state. This is a common situation in the outpatient department of our hospital. As a result, physicians have to assess cholesterol control using non-fasting measurement of blood lipids. According to the joint consensus statement of European Atherosclerosis Society and European Federation of Clinical Chemistry and Laboratory Medicine [9], the non-fasting detection of blood lipids can be routinely applied in CHD patients as long as they are willing to undergo non-fasting measurement. This suggests that measurement of LDL-C level in the non-fasting state is quite important.

Compared with some studies with large population in other countries $[14,15,17,18]$, the reduction in LDL$\mathrm{C}$ level in CHD patients after a daily meal was more significant in the present study. The maximum mean reduction in LDL-C or non-HDL-C was approximately $0.1-0.2 \mathrm{mmol} / \mathrm{L}$ in the European and North American subjects $[14,15,17,18]$; however, Chinese CHD patients in this study showed a greater decrease in either directly detected LDL-C (i.e., $0.4-0.5 \mathrm{mmol} / \mathrm{L}$ ) or calculated non-HDL-C (i.e., $0.2-0.3 \mathrm{mmol} / \mathrm{L}$ ) after a daily breakfast. Although our recent study showed that the postprandial decline (i.e., 0.3$0.4 \mathrm{mmol} / \mathrm{L}$ ) in calculated LDL-C was less than that of the directly detected LDL at $2-4 \mathrm{~h}$ after a daily breakfast in Chinese CHD patients [13], it was still more than the reduction of above the European and North American studies $[14,15,17,18]$. The underling mechanisms of non-fasting reduction in LDL-C may be complicated in the present study. First, in the Copenhagen General Population Study, they compared blood lipids levels of individuals at random time points after the last meal in the large-scale population. By contrast, our measurements were acquired from the same individuals at various times since the last meal, which was different from the Copenhagen General Population Study in terms of the observation time-points and monitoring method. Second, postprandial reduction in LDL-C concentration is most likely haemodilution resulting from fluid intake in relation to the meal and thus adjusting the data for albumin concentration was recommended [11, 14]. Langsted et al. [14] observed that the non-fasting LDL-C concentration no longer changed after adjustment for albumin concentration. However, a very slight change in the postprandial albumin level was observed in our study; therefore, haemodilution may not be the only cause of postprandial decline in the LDL-C level in the Chinese. Third, the diet structures of Chinese and western people are very different. For example, the Chinese people prefer carbohydrates [16]. 
It is not clear whether the high-carbohydrate diet will cause a more significant decline in cholesterol. At any rate, the obvious decrease in non-fasting LDL-C might affect the evaluation of goal attainment when the LDL-C level was detected after a meal.

Indeed, it was found that the percent attainment of postprandial LDL-C or/and non-HDL-C was significantly higher than that of fasting values in the present study, suggesting that the fasting goals of LDL-C $<1.8 \mathrm{mmol} / \mathrm{L}$ and non- HDL-C $<2.6 \mathrm{mmol} / \mathrm{L}$ were indeed unsuitable for the evaluation of postprandial cholesterol control. ROC analysis has been used to identify the optimal cut-off point for the diagnosis of postprandial hypertriglyceridemia $[16,28,29]$ but not for determining goals of LDL-C and non-HDL-C in the non-fasting state corresponding to the fasting goals. Because the non-fasting cut-off points acquired by ROC analysis corresponded to the fasting goals of LDL-C $<1.8 \mathrm{mmol} / \mathrm{L}$ and non-HDL$\mathrm{C}<2.6 \mathrm{mmol} / \mathrm{L}$, the postprandial percent attainments were very similar to their respective fasting values. This suggested that lower postprandial cut-off points, different from thee fasting goals, should be adopted in the evaluation of postprandial goal attainment, unless it is possible to assess the percentage reduction in the non-fasting LDL-C level.

Non-HDL-C represents the cholesterol content of all atherosclerotic lipoproteins in the circulation, including chylomicrons, very-low-density-lipid and their remnants, intermediate-density lipoproteins, LDL, and lipoprotein (a) particles. Takahiro found that non-HDL cholesterol levels were clearly associated with future mortality and were less affected by fasting status or serum triglyceride levels [30]. Meta-analyses and prospective studies with large populations supported the opinion that on-treatment levels of nonHDL-C were stronger than that of LDL-C for future CVD risk estimation $[20,21]$. Therefore, some scholars proposed that the clinical benefit obtained from controlling non-HDL-C would be greater than the one obtained from controlling LDL-C [19-21, 23]. In this study, the non-fasting reduction in non-HDL-C was less than that in LDL-C and the difference between fasting and non-fasting percentage attainments of non-HDL-C $<2.6 \mathrm{mmol} / \mathrm{L}$ was less than that of $\mathrm{LDL}-\mathrm{C}<1.8 \mathrm{mmol} / \mathrm{L}$. This appears to support the superiority of non-HDL-C in contrast to LDL-C in the lipid-lowering evaluation after a daily meal. Nevertheless, the percent attainment of non-HDL-C was higher than that of LDL-C in both fasting and non-fasting states according to the goals of $\mathrm{LDL}-\mathrm{C}<1.8 \mathrm{mmol} / \mathrm{L}$ and non-HDL-C $<2.6 \mathrm{mmol} / \mathrm{L}$, respectively, in this study. Overestimation of the target attainment of cholesterol control after treatment could occur if only the non-HDL-C level was evaluated.

The difference between non-HDL-C and LDL-C will increase with TG elevation, which could exert a substantial influence on evaluation of cholesterol-lowering treatment [31, 32]. The fixed difference between fasting non-HDL-C and LDL-C goals was $30 \mathrm{mg} / \mathrm{dl}$ (i.e., $0.8 \mathrm{mmol} / \mathrm{L}$ ) when fasting TG level was $1.7 \mathrm{mmol} / \mathrm{L}$, reflecting the fact that cholesterol content within TG-rich lipoproteins was about 1.7/2.2 $0.8 \mathrm{mmol} / \mathrm{L}$. Some scholars found that the goal attainment of non-HDL-C was higher than that of LDL-C when fasting $T G$ was $<1.7 \mathrm{mmol} / \mathrm{L}$, while it was less than that of $L D L-C$ when fasting $T G>2.3 \mathrm{mmol} / \mathrm{L}$ [31]. This is consistent with our findings that the percent attainment of LDL-C $<1.8 \mathrm{mmol} / \mathrm{L}$ was significantly lower than that of non-HDL-C $<2.6 \mathrm{mmol} / \mathrm{L}$ in the fasting state; however, the difference in percent attainment between LDL-C and non-HDL-C after a daily breakfast became smaller with the 
increase in non-fasting TG level. Other scholars reported that the specific and fixed goals as non-HDL-C $0.8 \mathrm{mmol} / \mathrm{L}(30 \mathrm{mg} / \mathrm{dL}$ ) higher than the corresponding LDL-C goals were not sufficient for Chinese patients with $\mathrm{CHD}$ and proposed that flexible goals basing on TG level were more appropriate [32]. In this study, the difference $(1.5 \mathrm{mmol} / \mathrm{L}$ vs. $2.4 \mathrm{mmol} / \mathrm{L})$ between non-fasting cut-off points of LDL-C and non$\mathrm{HDL}-\mathrm{C}$ was $0.9 \mathrm{mmol} / \mathrm{L}$ corresponding to non-fasting TG level of approximately $2.0 \mathrm{mmol} / \mathrm{L}$ (i.e. $0.9 \times 2.2$ $=1.98 \approx 2.0$ ). This suggests that a larger difference between LDL-C and non-HDL-C should be considered in the evaluation of non-fasting goal attainment even after a daily meal without high fat.

This study had some limitations. First, it was a single centre study with a small sample size of inpatients but not outpatients. In the future, the suitability of non-fasting cut-off points in a large sample of arteriosclerotic cardiovascular disease patients, including patients with ischemic stroke and peripheral vascular disease, is worth exploring. Second, only the percent attainment of the goal, but not percentage reduction of LDL-C, was evaluated because of the lack of baseline levels of blood lipids. Third, according to the recommendation of the 2019 European guidelines [27], more patients with fasting LDL-C < $1.4 \mathrm{mmol} / \mathrm{L}$ should be enrolled in CHD2 group to determine a stricter non-fasting cut-off point through ROC analysis in further study.

\section{Conclusion}

In conclusion, lower non-fasting cut-off points are needed to evaluate the efficacy of cholesterol-lowering therapy after a daily meal if the percentage reduction of LDL-C cannot be evaluated.

\section{Abbreviations}

CHD: coronary heart disease; TG: triglyceride; TC: total cholesterol; LDL-C: low-density lipoprotein cholesterol; HDL-C: high-density lipoprotein cholesterol; Non-HDL-C: non-high-density lipoprotein cholesterol; ROC curve: receiver operating characteristic

\section{Declarations}

\section{Ethics approval and consent to participate}

The study was approved by the Ethics Committee of the Second Xiangya Hospital of Central South University and informed consent was gained from all participants.

\section{Consent for publication}

Not applicable

\section{Availability of data and material}

The datasets analyzed during the current study are available from the corresponding 
author on reasonable request

\section{Competing Interests}

The authors declare that they have no competing interests.

\section{Funding}

This study was supported by National Natural Science Foundation of China [grant numbers 81270956 , 81470577].

\section{Authors' contributions}

Li-Ling Guo, Yan-qiao Chen and Ling Liu carried out the experimental work and the data collection and interpretation. Qiu-zhen Lin, Feng Tian, Qun-Yan Xiang and Li-yuan Zhu participated in the design and coordination of experimental work, and acquisition of data. Ling Liu participated in the study design, data collection, analysis of data and preparation of the manuscript. Li-Ling Guo and Ling Liu carried out the study design, the analysis and interpretation of data and drafted the manuscript. All authors read and approved the final manuscript

\section{Acknowledgments}

Not applicable

\section{References}

1. Stone NJ, Robinson JG, Lichtenstein AH, Bairey Merz CN, Blum CB, Eckel RH, Goldberg AC, Gordon D, Levy D, Lloyd-Jones DM, et al: 2013 ACC/AHA guideline on the treatment of blood cholesterol to reduce atherosclerotic cardiovascular risk in adults: a report of the American College of Cardiology/American Heart Association Task Force on Practice Guidelines. J Am Coll Cardiol 2014, 63:2889-2934.

2. Joint committee for guideline r: 2016 Chinese guidelines for the management of dyslipidemia in adults. J Geriatr Cardiol 2018, 15:1-29.

3. Catapano AL, Graham I, De Backer G, Wiklund O, Chapman MJ, Drexel H, Hoes AW, Jennings CS, Landmesser U, Pedersen TR, et al: 2016 ESC/EAS Guidelines for the Management of Dyslipidaemias. Eur Heart J 2, 2016, 37:2999-3058.

4. Grundy SM, Stone NJ, Bailey AL, Beam C, Birtcher KK, Blumenthal RS, Braun LT, de Ferranti S, FaiellaTommasino J, Forman DE, et al: 2018 AHA/ACC/AACVPR/AAPA/ABC/ACPM/ADA/AGS/APhA/ASPC/NLA/PCNA Guideline on the Management of Blood Cholesterol: A Report of the American College of Cardiology/American Heart Association Task Force on Clinical Practice Guidelines. J Am Coll Cardiol 2019, 73:e285-e350. 
5. European Association for Cardiovascular P, Rehabilitation, Reiner Z, Catapano AL, De Backer G, Graham I, Taskinen MR, Wiklund O, Agewall S, Alegria E, et al: ESC/EAS Guidelines for the management of dyslipidaemias: the Task Force for the management of dyslipidaemias of the European Society of Cardiology (ESC) and the European Atherosclerosis Society (EAS). Eur Heart $J$ 2011, 32:1769-1818.

6. Pastromas S, Terzi AB, Tousoulis D, Koulouris S: Postprandial lipemia: an under-recognized atherogenic factor in patients with diabetes mellitus. Int J Cardiol 2008, 126:3-12.

7. Doran B, Guo Y, Xu J, Weintraub H, Mora S, Maron DJ, Bangalore S: Prognostic value of fasting versus nonfasting low-density lipoprotein cholesterol levels on long-term mortality: insight from the National Health and Nutrition Examination Survey III (NHANES-III). Circulation 2014, 130:546-553.

8. Langsted A, Nordestgaard BG: Nonfasting versus fasting lipid profile for cardiovascular risk prediction. Pathology 2019, 51:131-141.

9. Nordestgaard BG, Langsted A, Mora S, Kolovou G, Baum H, Bruckert E, Watts GF, Sypniewska G, Wiklund $\mathrm{O}$, Boren $\mathrm{J}$, et al: Fasting is not routinely required for determination of a lipid profile: clinical and laboratory implications including flagging at desirable concentration cut-points-a joint consensus statement from the European Atherosclerosis Society and European Federation of Clinical Chemistry and Laboratory Medicine. Eur Heart J 2016, 37:1944-1958.

10. National Clinical Guideline C: National Institute for Health and Clinical Excellence: Guidance. In Lipid Modification: Cardiovascular Risk Assessment and the Modification of Blood Lipids for the Primary and Secondary Prevention of Cardiovascular Disease. London: National Institute for Health and Care Excellence (UK) Copyright (c) National Clinical Guideline Centre, 2014.; 2014

11. Langsted A, Nordestgaard BG: Nonfasting lipids, lipoproteins, and apolipoproteins in individuals with and without diabetes: 58434 individuals from the Copenhagen General Population Study. Clin Chem 2011, 57:482-489.

12. Downs JR, O'Malley PG: Management of dyslipidemia for cardiovascular disease risk reduction: synopsis of the 2014 U.S. Department of Veterans Affairs and U.S. Department of Defense clinical practice guideline. Ann Intern Med 2015, 163:291-297.

13. Lin QZ, Chen YQ, Guo LL, Xiang QY, Tian F, Wen T, Liu L: Comparison of non-fasting LDL-C levels calculated by Friedewald formula with those directly measured in Chinese patients with coronary heart disease after a daily breakfast. Clin Chim Acta 2019, 495:399-405.

14. Langsted A, Freiberg JJ, Nordestgaard BG: Fasting and nonfasting lipid levels: influence of normal food intake on lipids, lipoproteins, apolipoproteins, and cardiovascular risk prediction. Circulation 2008, 118:2047-2056.

15. Mora S, Rifai N, Buring JE, Ridker PM: Fasting compared with nonfasting lipids and apolipoproteins for predicting incident cardiovascular events. Circulation 2008, 118:993-1001.

16. Tian F, Xiang QY, Zhang MY, Chen YQ, Lin QZ, Wen T, Liu L: Changes in non-fasting concentrations of blood lipids after a daily Chinese breakfast in overweight subjects without fasting hypertriglyceridemia. Clin Chim Acta 2019, 490:147-153. 
17. Sidhu D, Naugler C: Fasting time and lipid levels in a community-based population: a cross-sectional study. Arch Intern Med 2012, 172:1707-1710.

18. Bansal S, Buring JE, Rifai N, Mora S, Sacks FM, Ridker PM: Fasting compared with nonfasting triglycerides and risk of cardiovascular events in women. Jama 2007, 298:309-316.

19. Benn M: Apolipoprotein B levels, APOB alleles, and risk of ischemic cardiovascular disease in the general population, a review. Atherosclerosis 2009, 206:17-30.

20. Toth PP: Association of LDL Cholesterol, Non-HDL Cholesterol, and Apolipoprotein B Levels With Risk of Cardiovascular Events Among Patients Treated With Statins: A Meta-analysis. Yearbook of Endocrinology 2012, 2012:65-68.

21. Ridker PM, Rifai N, Cook NR, Bradwin G, Buring JE: Non-HDL cholesterol, apolipoproteins A-I and B100, standard lipid measures, lipid ratios, and CRP as risk factors for cardiovascular disease in women. Jama 2005, 294:326-333.

22. Di Angelantonio E, Gao P, Pennells L, Kaptoge S, Caslake M, Thompson A, Butterworth AS, Sarwar N, Wormser D, Saleheen D, et al: Lipid-related markers and cardiovascular disease prediction. Jama 2012, 307:2499-2506.

23. Carbayo Herencia JA, Simarro Rueda M, Palazon Bru A, Molina Escribano F, Ponce Garcia I, Artigao Rodenas LM, Caldevilla Bernardo D, Divison Garrote JA, Gil Guillen VF: Evaluation of non-HDL cholesterol as a predictor of non-fatal cardiovascular events in a prospective population cohort. Clin Investig Arterioscler 2018, 30:64-71.

24. de Vries M, Klop B, Castro Cabezas M: The use of the non-fasting lipid profile for lipid-lowering therapy in clinical practice - point of view. Atherosclerosis 2014, 234:473-475.

25. Zhao Y, Peng R, Zhao W, Liu Q, Guo Y, Zhao S, Xu D: Zhibitai and low-dose atorvastatin reduce blood lipids and inflammation in patients with coronary artery disease. Medicine (Baltimore) 2017, 96:e6104.

26. Tian F, Wu CL, Yu BL, Liu L, Hu JR: Apolipoprotein O expression in mouse liver enhances hepatic lipid accumulation by impairing mitochondrial function. Biochem Biophys Res Commun 2017, 491:8-14.

27. Mach F, Baigent C, Catapano AL, Koskinas KC, Casula M, Badimon L, Chapman MJ, De Backer GG, Delgado V, Ference BA, et al: 2019 ESC/EAS Guidelines for the management of dyslipidaemias: lipid modification to reduce cardiovascular risk. Eur Heart J 2019.

28. White KT, Moorthy MV, Akinkuolie AO, Demler O, Ridker PM, Cook NR, Mora S: Identifying an Optimal Cutpoint for the Diagnosis of Hypertriglyceridemia in the Nonfasting State. Clin Chem 2015, 61:11561163.

29. Sevilla-Gonzalez MDR, Aguilar-Salinas CA, Munoz-Hernandez L, Almeda-Valdes P, Mehta R, Zubiran R, Bello-Chavolla OY, Gomez-Velasco DV, Vargas-Vazquez A, Viveros-Ruiz T, et al: Identification of a threshold to discriminate fasting hypertriglyceridemia with postprandial values. Lipids Health Dis 2018, 17:156.

30. Ito T, Arima H, Fujiyoshi A, Miura K, Takashima N, Ohkubo T, Kadota A, Hayakawa T, Kita Y, Miyagawa $\mathrm{N}$, et al: Relationship between non-high-density lipoprotein cholesterol and the long-term mortality of 
cardiovascular diseases: NIPPON DATA 90. Int J Cardiol 2016, 220:262-267.

31. Al-Hashmi K, Al-Zakwani I, Al Mahmeed W, Arafah M, Al-Hinai AT, Shehab A, Al Tamimi O, Al Awadhi M, Al Herz S, Al Anazi F, et al: Non-high-density lipoprotein cholesterol target achievement in patients on lipid-lowering drugs and stratified by triglyceride levels in the Arabian Gulf. J Clin Lipidol 2016, 10:368-377.

32. Su X, Luo M, Tang X, Luo Y, Zheng X, Peng D, Investigators C-A: Goals of non-high density lipoprotein cholesterol need to be adjusted in Chinese acute coronary syndrome patients: Findings from the CCC-ACS project. Clin Chim Acta 2019, 496:48-54.

\section{Figures}
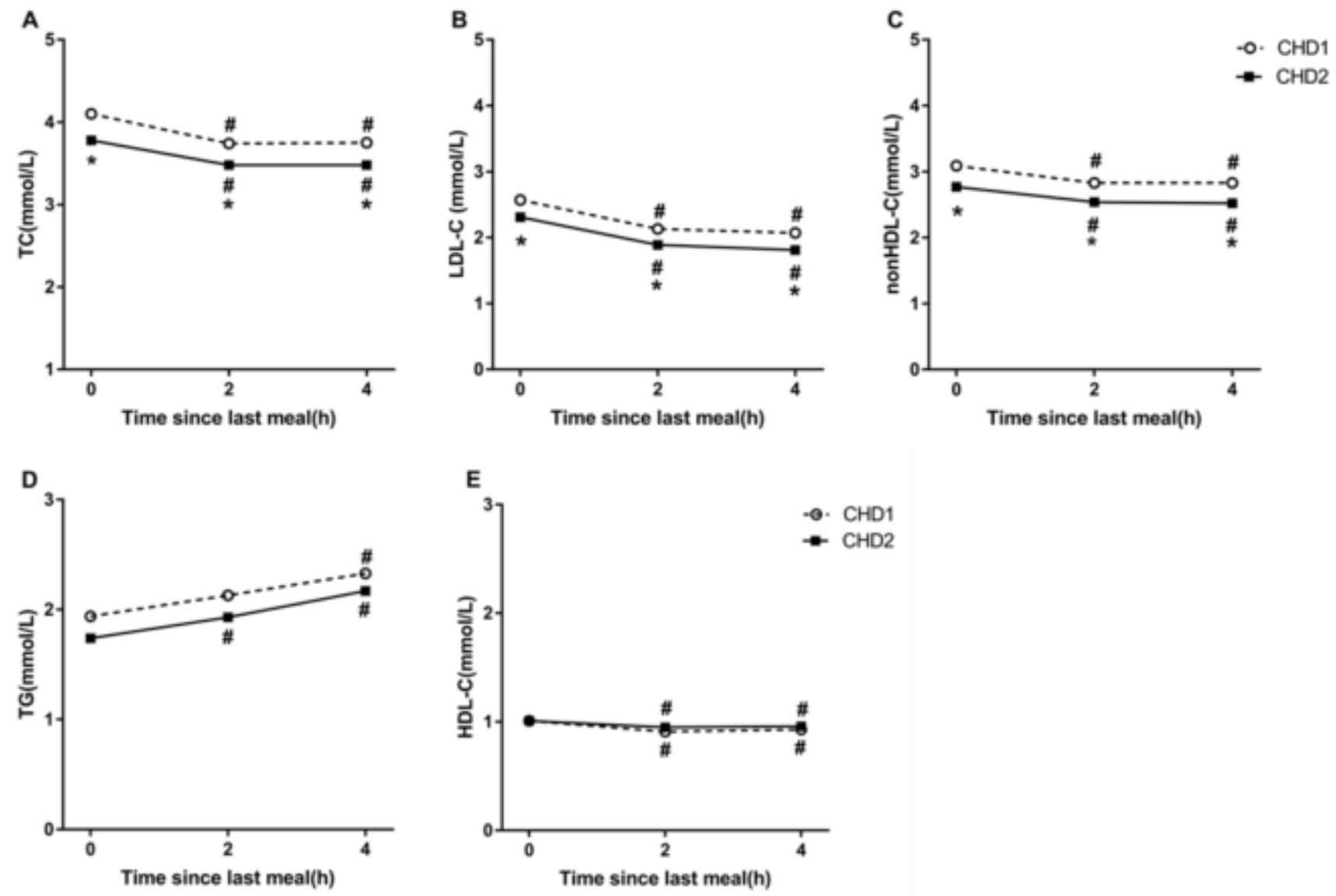

\section{Figure 1}

Comparison of changes in non-fasting blood lipids in two groups. Non-fasting changes in serum levels of TC (A), LDL-C (B), non-HDL-C (C), TG (D) and HDL-C (E). CHD1 group: CHD patients taking stains $<1 \mathrm{~m}$ and without stains treatment before admission. CHD2 group: CHD patients taking stains $\geq 1 \mathrm{~m}$ before admission. Values are means with SE. \# P 0.05 when compared with the fasting level in the same group. * $\mathrm{P}<0.05$ when compared with $\mathrm{CHD} 1$ group at the same time point. 

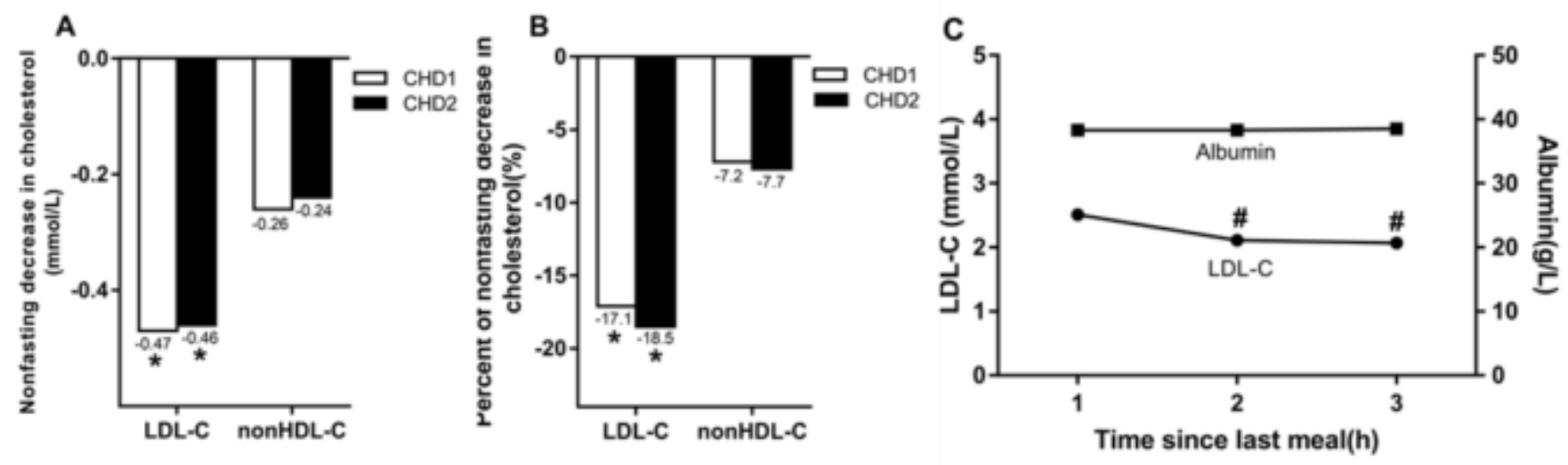

Figure 2

Non-fasting reductions in LDL-C and non-HDL-C levels in two groups. (A) Comparison of non-fasting absolute reductions in LDL-C and non-HDL-C levels. (B) Comparison of non-fasting percentages of reductions in LDL-C and non-HDL-C levels. CHD patients including patients in CHD1 group and CHD2 group. (C) Levels of LDL-C and albumin as a function of time since breakfast in 89 CHD patients. Values are means with SE. * $\mathrm{P}<0.05$ when compared with the absolute reduction or percentage of reduction in non-HDL-C level. \# P $<0.05$ when compared with the fasting level. CHD1 group: CHD patients taking stains $<1 \mathrm{~m}$ and without stains treatment before admission. CHD2 group: CHD patients taking stains $\geq 1 \mathrm{~m}$ before admission. 


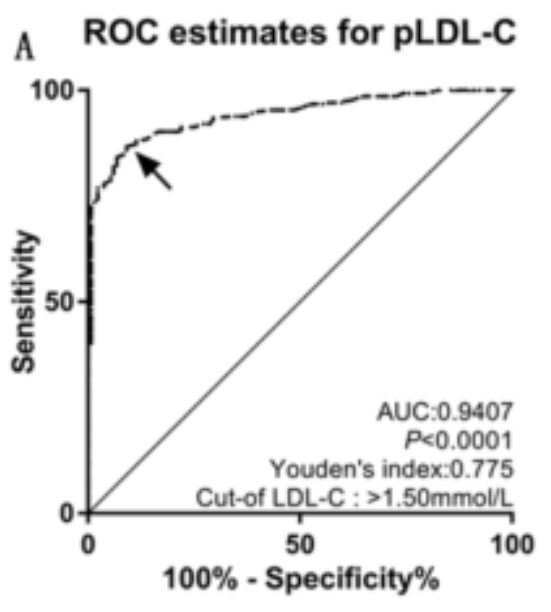

B ROC estimates for pnonHDL-C

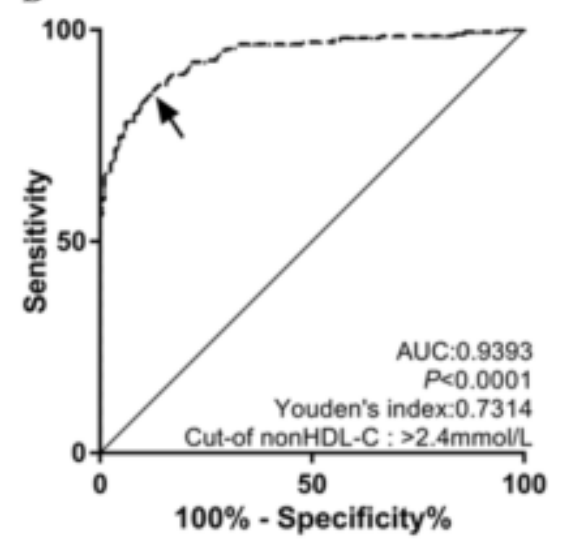

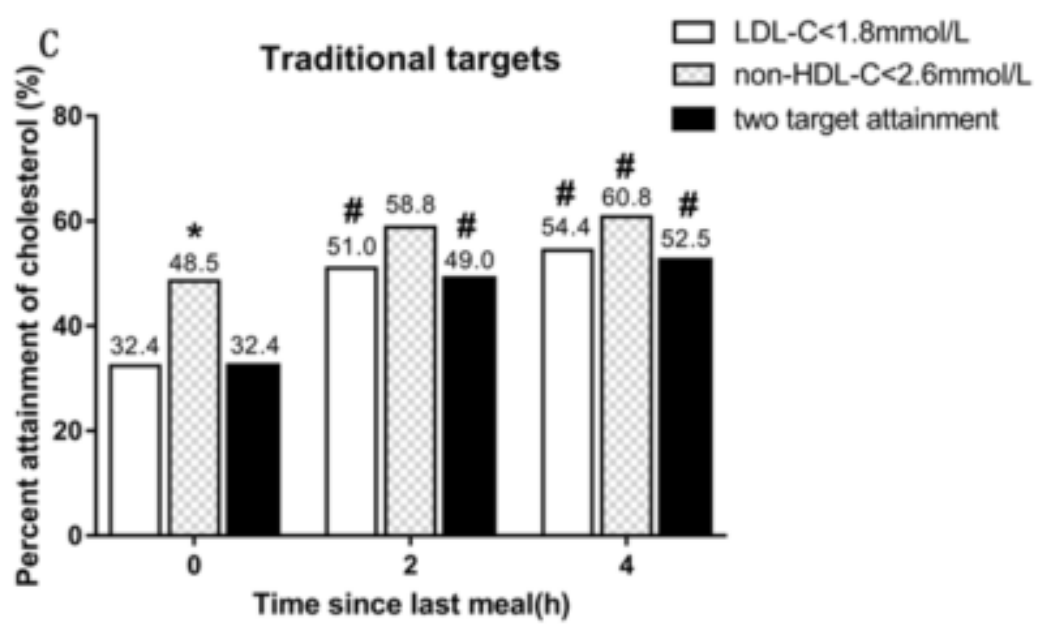

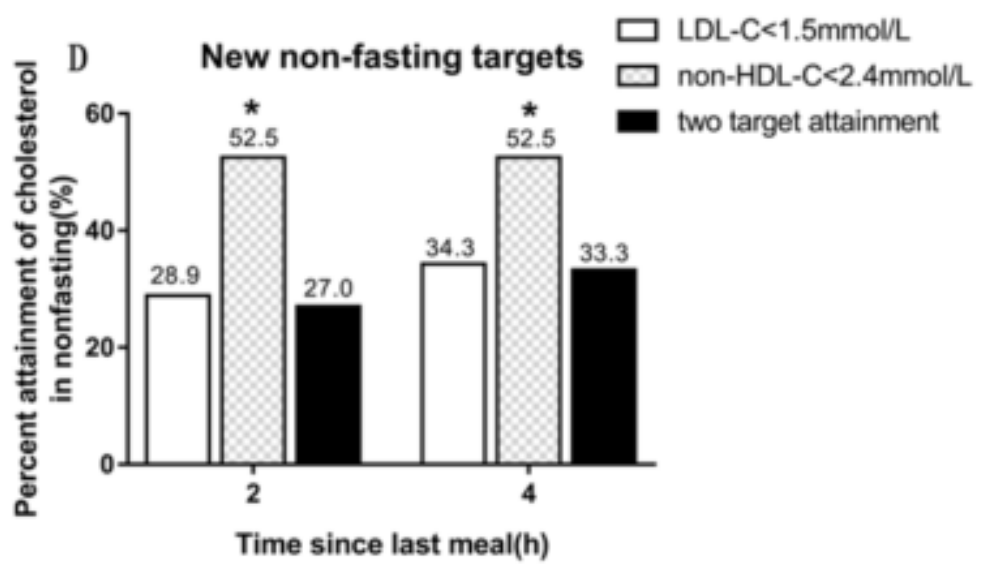

Figure 3

Comparison of the percent attainments of LDL-C and non-HDL-C levels according to different targets in fasting and non-fasting states in Group CHD2. CHD2 group: CHD patients taking stains $\geq 1 \mathrm{~m}$ before admission. (A, B) Non-fasting cut-off points in relation to fasting LDL-C level $1.8 \mathrm{mmol} / \mathrm{L}(\mathrm{A})$ and nonHDL-C level 2.6mmol/L (B) determined by ROC analysis and Youden's index (marked by the solid arrows).

(C) Comparison of the percent attainments in both fasting and non-fasting states according to the recommended targets of LDL-C level $<1.8 \mathrm{mmol} / \mathrm{L}$ and non-HDL-C level< $2.6 \mathrm{mmol} / \mathrm{L}$. (D) Comparison of the percent attainments according to non-fasting cut-off points of LDL-C $<1.5 \mathrm{mmol} / \mathrm{L}$ and non-HDL-C $<$ $2.4 \mathrm{mmol} / \mathrm{L}$. \# $\mathrm{P}<0.05$ when compared with the percent attainment of the same target(s) in the fasting state. ${ }^{*} P<0.05$ when compared with the percent attainment of different LDL-C target at the same time point.

\section{Supplementary Files}

This is a list of supplementary files associated with this preprint. Click to download.

- sumpplementarymaterial.docx 